\title{
On the nonexistence of conformally flat slices in the Kerr and other stationary spacetimes
}

\author{
Juan Antonio Valiente Kroon* \\ Institut für Theoretische Physik der Universität Wien, Boltzmanngasse 5, 1090 Wien, Austria.
}

(Dated: October 31, 2018)

\begin{abstract}
It is proved that a stationary solutions to the vacuum Einstein field equations with non-vanishing angular momentum have no Cauchy slice that is maximal, conformally flat, and non-boosted. The proof is based on results coming from a certain type of asymptotic expansions near null and spatial infinity - which also show that the developments of Bowen-York type of data cannot have a development admitting a smooth null infinity_- and from the fact that stationary solutions do admit a smooth null infinity.
\end{abstract}

PACS numbers: 04.20.Ex, 04.20.Ha, 04.70.Bw, 02.70.Wz

Introduction.- One of the basic tenets of what R. Penrose has called the establishment's view concerning the Physics of black holes, is that an asymptotically flat spacetime containing black holes will eventually settle down to a Kerr black hole. This point of view has been supported by the no hair theorems of Israel, Carter and Robinson [6, 16, 21], and confirmed by numerical simulations and perturbative analysis of the evolution of black hole initial data with non-vanishing angular momentum - see e.g. 1, 5, 10, 15. A great majority of these numerical simulations have made use of initial data sets which are conformally flat, and have a second fundamental form given by the so-called Bowen-York Ansatz [3]. As noted by several authors, the Bowen-York data do not reduce to Kerr data under any choice of the parameters. Now, there are several reasons why it would be of interest to have slices of the Kerr spacetime that are conformally flat. For example, one would like to analyse the evolution of a pair of initially very close spinning black holes as a perturbation of the Kerr solution, and not as a perturbation of Schwarzschild as it has been done so far - see e.g. 15.

Garat \& Price 14] have shown that the Kerr spacetime has no axially symmetric, conformally flat slices that smoothly approach to the standard Schwarzschildean slices of constant time as the angular momentum goes to zero. Their argument was based on a perturbative analysis of the Cotton-Bach tensor - which locally characterises conformally flat 3-dimensional hypersurfacesof the prospective slices up to second order in the angular momentum. Nevertheless, as noted by the authors themselves, there is, in principle, room for the existence more exotic slices than the ones covered by their Ansatz.

This letter presents a theorem that precludes the existence of maximal, non-boosted, conformally flat slices not only in the Kerr spacetime, but also in any stationary spacetime with non-vanishing angular momentum. The proof is based in general conformal properties of the stationary solutions to the Einstein field equations, and

*Electronic address: jav@ap.univie.ac.at makes use not only of the information contained in the slices, but also of features concerning their developments.

Let $\widetilde{S}$ be a Cauchy hypersurface, and let $\widetilde{h}_{\alpha \beta}, \widetilde{\chi}_{\alpha \beta}$ be, respectively, its first and second fundamental forms. We shall write $\tilde{\chi}=\tilde{\chi}_{\gamma}^{\gamma}$. The hypersurface $\widetilde{S}$ could have several asymptotically flat regions. We concentrate our attention on one of them. Let $\left\{y^{\alpha}\right\}$ be coordinates in this asymptotic region such that,

$\widetilde{h}_{\alpha \beta}=\left(1+\frac{2 m}{|y|}\right) \delta_{\alpha \beta}+\mathcal{O}\left(\frac{1}{|y|^{2}}\right), \quad \tilde{\chi}_{\alpha \beta}=\mathcal{O}\left(\frac{1}{|y|^{2}}\right)$,

as $|y| \rightarrow \infty$, where $m$ is the ADM mass associated with this end. The (ADM) linear and angular momentum of the asymptotically flat region are defined by,

$$
\begin{aligned}
& P_{\alpha}=\frac{1}{8 \pi} \lim _{r \rightarrow \infty} \int_{S_{r}}\left(\widetilde{\chi}_{\alpha \beta}-\tilde{\chi} \widetilde{h}_{\alpha \beta}\right) n^{\beta} d S_{r}, \\
& J_{\alpha}=\frac{1}{8 \pi} \lim _{r \rightarrow \infty} \int_{S_{r}} \epsilon_{\alpha \beta \gamma} y^{\beta}\left(\widetilde{\chi}^{\gamma \rho}-\tilde{\chi} \widetilde{h}^{\gamma \rho}\right) n_{\rho} d S_{r},
\end{aligned}
$$

respectively, where $S_{r}$ denotes the 2-sphere $\{|y|=r\}$, and $n^{\alpha}$ is the outward unit normal.

Our main theorem is the following:

Theorem. Let $\left(\widetilde{S}, \widetilde{h}_{\alpha \beta}, \widetilde{\chi}_{\alpha \beta}\right)$ be a maximal, asymptotically Euclidean, conformally flat initial data set for the Einstein vacuum field equations. Assume, also, that the initial data set contains no linear momentum, and that the conformally rescaled second fundamental form admits an expansion of the form given by equation (2). If, furthermore, the spacetime is stationary, then the angular momentum of the data must vanish.

Thus, stationary spacetimes -whence the Kerr solution - admit no maximal, conformally flat, nonboosted slices with a second fundamental form of the sort prescribed by equation (2) if their $\mathrm{ADM}$ angular momentum is non-zero. If the angular momentum were non-zero, then the Schwarzschild solution would render a counterexample to our claim.

Some remarks concerning the hypothesis of the theorem come into place. Firstly, the assumption of maximal 
slices - i.e. such that $\tilde{\chi}$ - is natural, for very little is know about how to solve the Einstein constraint equations for non-maximal data. Secondly, the slices have been assumed to be non-boosted, for the presence of linear momentum leads to non-smooth solutions of the Hamiltonian constraint - see [9]. Existence of solutions to the Hamiltonian constraint for a conformal second fundamental form of the sort given by equation (21) - under some extra conditions - has been discussed in the aforementioned reference.

Sketch of the proof: the proof of the theorem relies heavily on some conformal properties of stationary spacetimes and, in particular, on features of a certain kind of asymptotic expansions near spatial infinity that can be obtained from a representation of spatial infinity introduced by Friedrich [12]. For asymptotically Euclidean, conformally flat data with a second fundamental form like the one given in equation (2) —no stationarity being assumed here-, the asymptotic expansions contain certain logarithmic terms which preclude the existence of a smooth null infinity. These logarithmic terms vanish, if and only if, the angular momentum of the data vanishes. It can be seen that this implies that the development of such data admits no smooth null infinity. On the other hand, it is known that the developments of stationary data do admit a smooth null infinity. Consequently, if the initial data is to be stationary, its angular momentum must vanish.

There are, nevertheless, a number of technical issues that one has to analyse. These will be discussed in the sequel. In particular, there is the fact that the gauge in which the expansions near spatial and null infinity are obtained is different to that in which the existence of a smooth null infinity for stationary spacetimes has been proved. It could well be the case that the non-smoothness in the expansions is a product of a bad choice of gauge. As it turns out, this is not the case, and the way one can do it is by comparing them in a gauge well adapted to null infinity - the NP gauge.

Expansions near spatial and null infinity.- Penrose [19] has introduced the seminal idea of studying the spacetimes describing isolated systems by means of a manifold with boundary -the unphysical spacetime- $\left(M, g_{\mu \nu}\right)$. This unphysical spacetime is obtained from the original one, $\left(\widetilde{M}, \widetilde{g}_{\mu \nu}\right)$ by means of a conformal rescaling $g_{\mu \nu}=\Omega^{2} \widetilde{g}_{\mu \nu}$. The boundary of the unphysical spacetime consists, at least, of the so-called null infinity $\mathscr{I}=$ $\mathscr{I}^{+} \cup \mathscr{I}^{-}$, and of a point, $i^{0}$, called spatial infinity. The spacetime $\left(\widetilde{M}, \widetilde{g}_{\mu \nu}\right)$ will be said to admit a smooth null infinity, if a conformal compactification can be introduced in such a way that the resulting null infinity is a smooth submanifold.

Friedrich has introduced a certain representation of spatial infinity as a cylinder - the cylinder at spatial infinity, $I$ [12]. A major feature of this representation of null infinity, is that the location and structure of the conformal boundary of the spacetime is known a priori, if

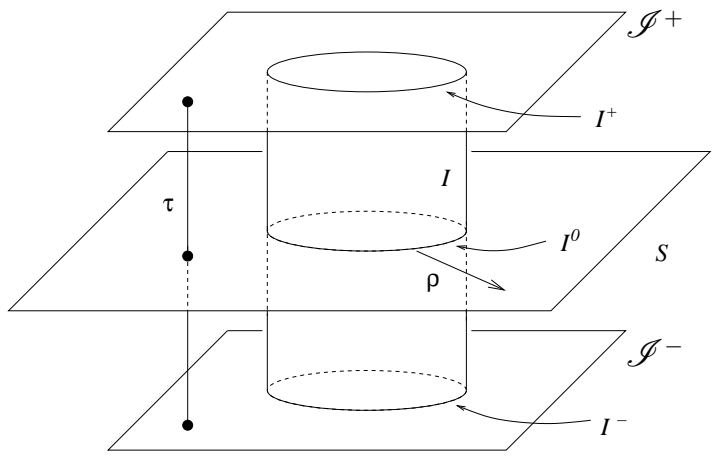

FIG. 1:

FIG. 2: The region of the - unphysical spacetime - near spatial and null infinities. The conformal factor leading to this representation is given by $\Theta=\omega \vartheta^{-2}\left(1-\tau^{2}\right)$ with $\omega=\vartheta /|d \vartheta|$.

the Einstein constraint equations have been solved. We shall refer to the gauge leading to this representation of spatial infinity as to the F-gauge. The equations governing the unphysical spacetime - the Conformal Einstein field equations - reduce, upon evaluation on $I$, to an interior system of transport equations. This system of transport equations enables us to obtain a certain type of asymptotic expansions, which in turn allow to connect properties of the spacetime at null infinity, with properties of the initial data. In particular, the components $\phi_{j}, j=0, \ldots, 4$ of the rescaled Weyl spinor $\phi_{a b c d}$ can be expanded as,

$$
\phi_{j} \sim \sum_{p \geq 0} \frac{1}{p !} \phi_{j}^{(p)}(\tau, \theta, \varphi) \rho^{p}
$$

$j=0, \ldots, 4$ where $\tau$ is a coordinate such that the locus of null infinity is given by $\tau= \pm 1$, and $(\theta, \varphi)$ are the standard polar coordinates. The cylinder at spatial infinity corresponds to the points for which $\rho=0$ and $-1 \leq \tau \leq 1$. The coefficients $\phi_{j}^{(p)}(\tau, \theta, \varphi)$ are determined by the interior system at $I$.

A set of scripts in the computer algebra system Maple $\mathrm{V}$ have been written in order to calculate the aforementioned expansions. They have been used to analyse the behaviour near spatial infinity of time symmetric solutions [22, 23, 25]. Recently, these scripts have been modified to enable the discussion of conformally flat data with non-vanishing second fundamental form. The full results of these investigations will be presented elsewhere. Here, we shall only mention the results which are relevant for our discussion.

Consider a conformally flat, maximal initial data set. Under the so-called conformal Ansatz, the constraint equations reduce to:

$$
\begin{aligned}
& \Delta \vartheta=-\frac{1}{8} \chi_{\alpha \beta} \chi^{\alpha \beta} \vartheta^{-7} \\
& \partial_{\alpha}\left(\vartheta^{4} \chi^{\alpha \beta}\right)=0
\end{aligned}
$$


where $\vartheta$ is the conformal factor linking with the conformal metric, $\delta_{\alpha \beta}=\vartheta^{-4} \widetilde{h}_{\alpha \beta}$. The conformally rescaled second fundamental form is given by $\chi_{\alpha \beta}=\vartheta^{-2} \widetilde{\chi}_{\alpha \beta}$. The conformal factor $\vartheta$ can be used to compactify the initial hypersurface $\widetilde{S}$. Let $x^{\alpha}$ denote normal coordinates such that $\rho=|x|=0$ at the point at infinity of the conformally rescaled initial hypersurface $S$.

In 9] Dain \& Friedrich have discussed a the existence of initial data for the Einstein vacuum equations which near spatial infinity admit expansions of the form,

$$
\widetilde{h}_{\alpha \beta} \sim\left(1+\frac{2 m}{r}\right) \delta_{\alpha \beta}+\sum_{k \geq 2} \frac{\widetilde{h}_{\alpha \beta}^{k}}{r^{k}}, \quad \tilde{\chi}_{\alpha \beta} \sim \sum_{k \geq 2} \frac{\widetilde{\chi}_{\alpha \beta}^{k}}{r^{k}},
$$

where the coefficients involved in the expansions are smooth functions on $S^{2}$. This type of data is precisely of the sort one needs in order to use the CA scripts we have previously discussed. The sufficient conditions they found for the existence of such data imply that in the conformally flat case the conformally rescaled second fundamental form has to be of the form,

$$
\chi_{\alpha \beta}=\chi_{\alpha \beta}^{A}+\chi_{\alpha \beta}^{J}+\chi_{\alpha \beta}^{Q}+\mathcal{O}(\rho),
$$

where

$$
\begin{aligned}
\chi_{\alpha \beta}^{A} & =\frac{A}{\rho^{3}}\left(3 n_{\alpha} n_{\beta}-\delta_{\alpha \beta}\right), \\
\chi_{\alpha \beta}^{J} & =\frac{3}{\rho^{3}}\left(n_{\beta} \epsilon_{\gamma \alpha \rho} J^{\rho} n^{\gamma}+n_{\alpha} \epsilon_{\rho \beta \gamma} J^{\gamma} n^{\rho}\right), \\
\chi_{\alpha \beta}^{Q} & =\frac{3}{2 \rho^{2}}\left(Q_{\alpha} n_{\beta}+Q_{\beta} n_{\alpha}-\left(\delta_{\alpha \beta}-n_{\alpha} n_{\beta}\right) Q^{\gamma} n_{\gamma}\right),
\end{aligned}
$$

and $n^{\alpha}=x^{\alpha} / \rho$ is the radial unit normal near infinity - see also [2]. The constants $A, J^{\alpha}, Q^{\alpha}$ are associated to the conformal Killing vectors on the Euclidean 3-dimensional space generating dilatations, rotations and "special conformal transformations" respectively.

For non-time symmetric data with a second fundamental form of the sort given in equation (2), the coefficients $\phi_{j}^{(0)}, \phi_{j}^{(1)}, \phi_{j}^{(2)}$, and $\phi_{j}^{(3)}$ in the expansion (1) are smooth functions of their arguments. However, for $\phi_{j}^{(4)}$ one has that,

$$
\phi_{j}^{(4)}=f_{j}^{\infty}+f_{j}^{-} \ln (1+\tau)+f_{j}^{+} \ln (1-\tau),
$$

where $f_{j}^{\infty}, f_{j}^{ \pm}$are analytic functions of $(\tau, \theta, \varphi)$. Furthermore, $f_{j}^{ \pm}$are polynomials in $\tau$-with coefficients depending on $(\theta, \varphi)$ - of the form,

$$
\begin{aligned}
& f_{0}^{+}(\tau)=m|J|^{4} P_{4}(\tau)(1-\tau)^{2}, \\
& f_{1}^{+}(\tau)=m|J|^{3} P_{3}(\tau)(1-\tau)^{3}, \\
& f_{2}^{+}(\tau)=m|J|^{2} P_{2}(\tau)(1-\tau)^{4}, \\
& f_{3}^{+}(\tau)=m|J|^{2} P_{1}(\tau)(1-\tau)^{5}, \\
& f_{4}^{+}(\tau)=m|J|^{2}(1-\tau)^{6},
\end{aligned}
$$

and $f_{j}^{-}(\tau)=f_{j}^{+}(-\tau)$. We have also written $|J|^{2}=J_{\alpha} J^{\alpha}$. The functions $P_{1}(\tau), \ldots, P_{4}(\tau)$ are polynomials in $\tau$ such that $P_{j}( \pm 1) \neq 0$. Thus, for conformally flat initial data, the presence of angular momentum is incompatible with a smooth null infinity. Note, that if the initial data is not conformally flat - as in the case of the standard Kerr data in Boyer-Lindquist coordinates - the situation is bound to be completely different. In this case the nonconformal parts of the metric would, in principle, leave room to cancel out the logarithmic singularities, although this must not be necessarily the case.

In order to compare the expansions implied by the aforementioned results, it is convenient to change our gauge from the F-gauge to the NP gauge. This is achieved by means of a conformal rescaling and a rotation of the tetrad in which the conformally rescaled Weyl spinor $\phi_{a b c d}$ is expressed. A full detailed list of what are the requirements for the NP gauge is given in 13]. After a lengthy calculation, it can be shown that the expansions (11) together with (3a)-(3e) imply in the NP gauge the following expansions for the components of the Weyl spinor, $\widetilde{\Psi}_{a b c d}$, of the physical (unrescaled) spacetime,

$$
\begin{aligned}
\widetilde{\Psi}_{0} & \sim k_{0} m|J|^{2} \Omega^{3} \ln \Omega+\widetilde{\Psi}_{0}^{3} \Omega^{3} \cdots, \\
\widetilde{\Psi}_{1} & \sim k_{1} m|J|^{2} \Omega^{3} \ln \Omega+\widetilde{\Psi}_{1}^{3} \Omega^{3} \cdots, \\
\widetilde{\Psi}_{2} & \sim k_{2} m|J|^{2} \Omega^{3} \ln \Omega+\widetilde{\Psi}_{2}^{3} \Omega^{3} \cdots, \\
\widetilde{\Psi}_{3} & \sim \widetilde{\Psi}_{3}^{2} \Omega^{2}+\cdots, \\
\widetilde{\Psi}_{4} & \sim \widetilde{\Psi}_{4}^{1} \Omega+\cdots .
\end{aligned}
$$

Here $k_{0}, k_{1}$ and $k_{2}$ denote non-zero constants, while the coefficients $\widetilde{\Psi}_{j}^{n}$ are functions of a retarded time $u$, which is defined up to a supertranslation, and the angular coordinates $(\theta, \varphi)$. Thus, note that if the angular momentum does not vanish, then null infinity not only is non-smooth, but the Peeling behaviour is not satisfied. It is noted that a somewhat similar result has been obtained by Klainerman and Nicolò - cfr. the remark of theorem 1.2 in [17]. It is also noted that the quantity $m|J|^{2}$ is essentially the constants associated to the leading logarithmic terms which have been discussed in 7, 24]. The details of this, and a further connection to the so-called logarithmic Newman-Penrose constants will be also discussed elsewhere.

It is also noted that the above expansions imply that the development of Bowen-York type data with nonvanishing $\mathrm{ADM}$ angular momentum does not admit a smooth null infinity. This statement holds for the data constructed by Brandt \& Brügmann [4] as they are also conformally flat and use the same Ansatz for the second fundamental form as the considered by Bowen \& York.

The stationary solutions near null infinity.- That the stationary solutions can be analytically extended through null infinity is known from the appendix in 11] and the results of [8]. Now, the metric given in [11] is not, in principle, in the NP gauge. Consider a null frame 
$\left\{l^{\mu}, n^{\mu}, m^{\mu}, \bar{m}^{\mu}\right\}$ for that metric. A lengthy, but straightforward argument shows that the coordinate transformations and the Lorentz transformations required to take Damour \& Schmidt's metric to the NP gauge are analytic functions. Indeed, one has the following,

Lemma. The stationary solutions written in the NP gauge have an analytic null infinity.

Moreover, it can be shown that — see e.g. [18, 20]-,

$$
\begin{aligned}
& \widetilde{\Psi}_{0}=\widetilde{\Psi}_{0}^{5} \Omega^{5}+\mathcal{O}\left(\Omega^{6}\right), \\
& \widetilde{\Psi}_{1}=\widetilde{\Psi}_{1}^{4} \Omega^{4}+\mathcal{O}\left(\Omega^{5}\right), \\
& \widetilde{\Psi}_{2}=-m \Omega^{3}+\mathcal{O}\left(\Omega^{4}\right), \\
& \widetilde{\Psi}_{3}=\mathcal{O}\left(\Omega^{3}\right), \\
& \widetilde{\Psi}_{4}=\mathcal{O}\left(\Omega^{3}\right),
\end{aligned}
$$

where the coefficients $\widetilde{\Psi}_{j}^{n}$ are $u$-independent.

Thus, one sees that if the further requirement of stationarity is imposed on the expansions (4a)-(4e) then necessarily $|J|^{2}=0$.
The only property of the stationary solutions that has been used is that they admit a smooth null infinity. This may look at first sight rather odd. However the results obtained in 22, 23] and the ones discussed here suggest that admitting a smooth null infinity near spatial infinity may be an alternative characterisation of the stationary spacetimes. This, nevertheless remains to be proved.

\section{Acknowledgments}

I would like to thank R. Beig, M. Mars, S. Dain and R. Lazkoz for very helpful discussions and suggestions. This project is funded by a Lise Meitner fellowship (M690N09) of the Fonds zur Forderung der Wissenschaftlichen Forschung - FWF- Austria. The CA calculations described have been carried out in the computers of the Max Planck Institut für Gravitationsphysk, Albert Einstein Institut, Golm bei Potsdam, Germany.
[1] J. Baker, B. Brügmann, M. Campanelli, C. O. Lousto, \& R. Takahashi, Plunge waveforms from inspiralling binary black holes, Phys. Rev. Lett. 87, 121103 (2001).

[2] R. Beig \& N. O'Murchadha, The momentum constraints of General Relativity and spatial conformal isometries, Comm. Math. Phys. 176, 723 (1996).

[3] J. M. Bowen \& J. W. York Jr., Time-asymmetric initial data for black holes and black-hole collsions, Phys. Rev. D 21, 2047 (1980).

[4] S. Brandt \& B. Brügmann, A simple construction of initial data for multiple black holes, Phys. Rev. Lett. 78, 3606 (1997).

[5] S. R. Brandt \& E. Seidel, Evolution of distorted black holes. I. Methods and tests, Phys. Rev. D 52, 856 (1995).

[6] B. Carter, Axisymmetry black hole has only two degrees of freedom, Phys. Rev. Lett. 26, 331 (1971).

[7] P. T. Chruściel, M. A. H. MacCallum, \& D. B. Singleton, Gravitational waves in general relativity XIV. Bondi expansions and the "polyhomogeneity" of $\mathscr{I}$, Phil. Trans. Roy. Soc. Lond. A 350, 113 (1995).

[8] S. Dain, Initial data for stationary spacetimes near spacelike infinity, Class. Quantum Grav. 18, 4329 (2001).

[9] S. Dain \& H. Friedrich, Asymptotically flat initial data with prescribed regularity at infinity, Comm. Math. Phys. 222, 569 (2001).

[10] S. Dain, C. Lousto, \& R. Takahashi, New conformally flat initial data for spinning blackholes, Phys. Rev. D 65, 104038 (2002).

[11] T. Damour \& B. Schmidt, Reliability of perturbation theory in general relativity, J. Math. Phys. 31, 2441 (1990).

[12] H. Friedrich, Gravitational fields near space-like and null infinity, J. Geom. Phys. 24, 83 (1998).

[13] H. Friedrich \& J. Kánnár, Bondi-type systems near spacelike infinity and the calculation of the NP-constants, J.
Math. Phys. 41, 2195 (2000).

[14] A. Garat \& R. H. Price, Nonexistence of conformally flat slaices of the Kerr spacetime, Phys. Rev. D 61, 124011 (2000).

[15] R. J. Gleiser, C. O. Nicasio, R. H. Price, \& J. Pullin, Evolving the Bowen-York initial data for spinning black holes, Phys. Rev. D D57, 3401 (1998).

[16] W. Israel, Event horizons in static vacuum space-times, Phys. Rev. 164, 1776 (1967).

[17] S. Klainerman \& F. Nicolò, Peeling properties of asymptotically flat solutions to the Einstein vacuum equations, Class. Quantum Grav. 20, 3215 (2003).

[18] E. T. Newman \& R. Penrose, New conservation laws for zero rest-mass fields in asymptotically flat space-time, Proc. Roy. Soc. Lond. A 305, 175 (1968).

[19] R. Penrose, Asymptotic properties of fields and spacetimes, Phys. Rev. Lett. 10, 66 (1963).

[20] R. Penrose \& W. Rindler, Spinors and space-time. Volume 2. Spinor and twistor methods in space-time geometry, Cambridge University Press, 1986.

[21] D. C. Robinson, Uniqueness of the Kerr black hole, Phys. Rev. Lett. 34, 905 (1975).

[22] J. A. Valiente Kroon, Does asymptotic simplicity allow for radiation near spatial infinity?, in gr-qc/0309016

[23] J. A. Valiente Kroon, A new class of obstructions to the smoothness of null infinity, to appear in Comm. Math. Phys. Also in gr-qc/0211024

[24] J. A. Valiente Kroon, Logarithmic Newman-Penrose Constants for arbitrary polyhomogeneous spacetimes, Class. Quantum Grav. 16, 1653 (1999).

[25] J. A. Valiente Kroon, Early radiative properties of the developments of time symmetric conformally flat initial data, Class. Quantum Grav. 20, L53 (2003). 